\title{
Time-Series Transcriptome Sheds Light on Anthocyanin Metabolism and Species Diversification Between two Lonicera Japonica Thunb Cultivars
}

\author{
Jianjun Li ( $\sim 043081 @$ @tu.cn ) \\ Henan Normal University https://orcid.org/0000-0002-6034-2411 \\ Chenglin Ye \\ Henan Normal University \\ Jingxiao $\mathrm{Ma}$ \\ Henan Normal University \\ Ting Cheng \\ Henan Normal University \\ Yan LV \\ Henan Normal University \\ Cuifang Chang \\ Henan Normal University
}

\section{Research}

Keywords: Lonicera Japonica Thunb, Anthocyanin, Yujin 2, Comparative transcriptomics, RNA-seq

Posted Date: November 12th, 2020

DOl: https://doi.org/10.21203/rs.3.rs-104094/v1

License: (c) (i) This work is licensed under a Creative Commons Attribution 4.0 International License.

Read Full License 


\section{Abstract}

Background $\nabla^{\prime} Y$ ujin 2' is new variety of Lonicera japonica Thunb and its flower color can change from red to yellow; hence, it is a good model for investigating flower color development mechanisms.

Results囚High throughput transcriptome sequencing of seven flower development stages of Yujin No.2 was carried out, and 133,487 unigenes were annotated, among which 73,088 were differentially expressed. Then the real-time PCR analysis was carried out. Further, the number of up-regulated DEGs was higher than those that were down-regulated. Of these annotated DEGs, plant hormone signal transduction, phenylpropanoid biosynthesis, and flavonoid biosynthesis were active throughout the flowering process during each stage, whereas carotenoid biosynthesis was inactive in the 51-6 stages. Furthermore, phenylalanine synthesis was enhanced in the S1 phase; however, anthocyanin synthesis was weakened in the S5 and S6 phases, which may be consistent with the changes in petal color of 'Yujin 2 ' from red (S1) to white (S5) and gold (S6). The results showed that 114 unigenes were associated with anthocyanin metabolism, and 72 were significantly upregulated or downregulated. According to the analysis of TFs in anthocyanin metabolism, we obtained 47 transcription factors, which belonged to 18 families. The LjDFR, LjABCB1, LjMYC6, LjDDB2, and LjANS genes rapidly increased during the first three stages. However, only LjF3'5'H expression was significantly down-regulated at S5, which was consistent with anthocyanin accumulation.

Conclusions $₫$ This study developed a transcriptome profile of flower color generation for $L$. japonica as well as annotated unigene sets of seven anthesis phases, thereby providing possibilities for improving the germplasm of $L$. japonica with genetic engineering technologies and cultivating new varieties of different colors.

\section{Background}

Lonicera japonica Thunb (L. japonica) is a perennial semi-evergreen twining stoloniferous shrub.

Traditionally, the L. japonica flower bud, which has been listed in the Chinese Pharmacopoeia as Lonicera japonica (Jinyinhua in Chinese) is considered to be the primary medicinal component [1]. Recent studies have found that $L$. japonica has many medicinal properties due to its antioxidant [2], hypoglycemic and hypolipidemic [3], anti-allergic [4], anti-inflammatory [5], and antibacterial effects [6]. Further, the demand for $L$. japonica in pharmaceuticals [1], cosmetics [7], and health foods [8] has increased. Although $L$. japonica is widely distributed in China, its yields and quality are the highest in Fengqiu of Henan and Shandong Provinces. Therefore, our research group selected and bred a new variety (Yujin 2). It has the characteristics of red buds, strong resistance, and a high content of chlorogenic acid (CGA) and luteoloside. The red buds endow 'Yujin 2' with enhanced beauty, which is more valuable in utility [9].

As one of the most important phenotypic traits of ornamental plants, the formation of flower color is the result of the combined effect of environmental influences and genetic characteristics [10]. The modification of petal color regulation genes is indispensable in modern flower breeding [11]. Through 
various studies it has been established that changes in flower color are primarily due to alterations in plant pigment metabolism [12]. The key pigments to adjust the color are carotenoids, flavonoids, and alkaloids $[11 ; 13 ; 14]$.

Anthocyanins, which are produced through flavonoid biosynthesis pathways during plant metabolism, are important water-soluble natural food pigment, which is widely present in plant epidermal cell vacuoles [15]. In the first stage of anthocyanin biosynthesis, phenylalanine transforms into 4-coumaryl:CoA, referred to as the phenylpropanoid metabolic pathway. The next stage, 4-coumaryl:CoA transforms into multiple flavonoid compounds [16].

The biosynthesis of anthocyanins is related to a variety of structures and regulatory genes [17]. The expression of chalcone synthase (CHS), Flavanone 3-hydroxylase (F3H), dihydroflavonol-4-reductase (DFR), anthocyanin synthase (ANS) and UDP glucoflavonoid 3-o-glucosyltransferase (UFGT) directly affect the accumulation of anthocyanins in plants [18]. These structural genes have been cloned in different plants, and the expression pathway is common to different types of plants.

Key regulatory genes serve as regulators that combine individually, or in the form of dimers and trimers, which play a critical role in the regulation of anthocyanin synthesis. So far, MYB, bHLH and WD40 transcription factors are widely regarded as the three main regulatory factors [19]. The MYB family transcription factor is one of the largest transcription factor families in plants, which has a MYB domain that is composed of $1 \sim 4$ MYB repeating units in its structure [20]. In conjunction with MYB, the bHLH transcription factors play active and critical roles in anthocyanin biosynthesis. The high anthocyanin content of many crops results from the increased expression of bHLH transcription factors [10]. WD40 is an ancient and highly conserved family of proteins that is involved in myriad plant cellular processes, such as transcriptional regulation, cell division, vesicle formation and transport, processing, signal transduction and so on [21].

There were great differences in the corolla color of 'Yujin 2' at the various developmental stages. The upper half of the flower bud during the early stage was purple-red, whereas the whole flower bud from the three green periods to the great white period was red, but gradually became lighter; whereas during the silver period, the exterior was red and the interior was white. During the golden period, the exterior of ' $Y$ ujin 2 ' was red and the interior was white, but for 'Damaohua' it was yellow [9].

Color is considered to be an important indicator in the assessment of ornamental value and offers a basis for plant classification [11]. However, the molecular mechanisms of anthocyanin metabolism in $L$. japonica flowers have not yet been discovered. Therefore, this paper employed 'Yujin 2 and 'Damaohua' flowers at different stages of development to study the molecular mechanisms of anthocyanin formation. This, by means of morphology, transcriptome, and bioinformatics, etc., to provide possibilities for enhancing the $L$. japonica germplasm using genetic engineering technologies, and cultivating new varieties with different colors. 


\section{Results}

\section{Characteristics of 'Damaohua' and 'Yujin 2' transcriptome}

A total of 42 samples ('Damaohua' and 'Yujin 2', respectively, have seven stages, each with three independent replicates) were sequenced using the Illumina HiSeq X Ten sequencer (Illumina Inc., USA), from which $311 \mathrm{~Gb}$ of raw data was harvested. Using a pair-end sequencing strategy, $2 \mathrm{G}$ raw reads with mean lengths of $150 \mathrm{nt}$ were obtained from the 42 samples with $47.62 \mathrm{M}$ reads for each sample. After the filtering of raw reads, $1.95 \mathrm{G}$ reads were counted, with an average of $46.43 \mathrm{M}$ reads per sample (Additional file 1: Table S1). Following the removal of redundant sequences and quantity control, 133,487 unigenes were assembled. The average length of the unigenes was $1,047 \mathrm{bp}$ with $45.75 \% \mathrm{GC}$ and $1642 \mathrm{nt}$ N50. The longest transcript was 16,864 bp, and the shortest was $301 \mathrm{bp}$.

The unigenes were annotated to obtain functional information using seven separate databases, including NCBI non-redundant protein (NR), Clusters of Orthologous Groups (COG) for eukaryotic complete genomes, Gene Ontology (GO), Swiss-Prot, evolutionary genealogy of genes: Non-supervised Orthologous Groups (eggNOG), and the Kyoto Encyclopedia of Genes and Genomes (KEGG) databases by Diamond Software, and mapped to Pfam databases by HMMER. Overall, $66.79 \%$ (133,487 unigenes) were successfully annotated, among which most unigenes (66.46\%) were assigned to annotation terms in the NR database (Table 1). Further, $61.43 \%$ of the unigenes were annotated on eggNOG, $46.62 \%$ on Swissprot, $43.46 \%$ on GO, $35.76 \%$ on KOG, and $26.02 \%$ on the KEGG pathway (Table 1 ).

Table 1

Percentage of annotated unigenes.

\begin{tabular}{|ll|}
\hline Database & Count of annotated unigenes $\%$ \\
\hline NR & $66.46 \%$ \\
\hline KOG & $35.76 \%$ \\
GO & $43.46 \%$ \\
\hline Swiss-prot & $46.62 \%$ \\
eggNOG & $61.43 \%$ \\
KEGG & $26.02 \%$ \\
Pfam & $0.13 \%$ \\
\hline Total & $66.79 \%$ \\
\hline
\end{tabular}

According to the NR annotation results, the current study compared $L$. japonica with taxonomic species stored in the NR database. A total of 97247 sequences were matched with L. japonica unigenes, which covered 1512 taxonomy categories (Additional file 2: Table S2). The highest ranked species was 
Alternaria alternate, which took up $12.82 \%$, whereas the second highest-ranked was Vitis vinifera, which contributed $8.80 \%$ (Fig. 1).

\section{Differentially expressed genes (DEGs)}

When comparing seven flowering stages of expressed genes between 'Damaohua' and 'Yujin 2', 73,088 differentially expressed genes exhibited remarkable changes. A total of 5,355 genes were differentially expressed, with 2,864 genes up-regulated and 2491 genes down-regulated at the S1 stage. At the S2 stage, 21,159 genes were differentially expressed, with 12,734 genes up-regulated and 8,425 genes downregulated. At the S3 stage, 19,126 genes were differentially expressed, with 10,343 genes up-regulated and 8,783 genes down-regulated. At the $S 4$ stage, 11,153 genes were differentially expressed, with 6,029 genes up-regulated and 5,124 genes down-regulated. At the $S 5$ stage, 27,718 genes were differentially expressed, with 12,102 genes up-regulated and 15,616 genes down-regulated. At the S6 stage, 18,652 genes were differentially expressed, with 8,486 genes up-regulated and 10,166 genes down-regulated. Finally, at the S7 stage, 38,162 genes were differentially expressed, with 21,153 genes up-regulated and 17,009 genes down-regulated (Fig. 2). As shown in the figures, the number of up-regulated DEGs was higher than those that were down-regulated.

\section{KEGG-pathway analysis of differentially expressed genes}

Unigenes were assigned to KEGG pathways based on annotation data. DEGs were enriched into 181, 202, 202, 191, 207, 204, and 210 pathways (S1, S2, S3, S4, S5, S6, and S7) (Additional file 3: Table S3). At the S1 stage, 53 pathways were enriched with a statistically significant enrichment $p$-value. Plant hormone signal transduction (k004075), and phenylpropanoid biosynthesis (k000940) during the entire process, as well as up-regulation and down-regulation processes, played active roles, and the expression was significant. Amino sugar and nucleotide sugar metabolism (k00520) were also active during the entire process and up-regulation, while they were not active in the down-regulation process. Starch and sucrose metabolism (ko00500) were active during the entire process and down-regulation process, while they were not active in the up-regulation process (Figs. 1A).

At the S2 stage, 72 pathways were enriched with a statistically significant enrichment $p$-value, which was more than the other six paired comparison phases. Endocytosis (ko04144), plant hormone signal transduction (ko04075), and starch and sucrose metabolism (ko00500) during the entire process, as well as up- and down-regulation processes played an active role, and the expression was significant. Phenylpropanoid biosynthesis (ko00940) was active during the entire process and the down-regulation process, while it decreased in the up-regulation process (Figs. 1B).

At the S3 stage, 66 pathways were enriched with a statistically significant enrichment $p$-value. Plant hormone signal transduction (ko04075), phenylpropanoid biosynthesis (k000940), starch and sucrose metabolism (ko00500) during the entire process, and the up- and down-regulation processes played an active role, and the expression is significant. Glycolysis/Gluconeogenesis (ko00010) was active during 
the entire process and the down-regulation process, while it was not active in the up-regulation process (Figs. 1C).

At the S4 stage, 71 pathways were enriched with a statistically significant enrichment $p$-value, and plant hormone signal transduction (ko04075) during the entire process, the up- and down-regulation processes played an active role, and the expression was significant. Phenylpropanoid biosynthesis (ko00940) and cell cycle (ko04110) were active during the entire process and the up-regulation process, while they were not active in the down-regulation process. Starch and sucrose metabolism (ko00500) were active during the entire process and the down-regulation process, while they were not active in the up-regulation process. Cell cycle-yeast (ko04111) was triggered in the up-regulation process, while carbon metabolism (ko01200) was triggered in the down-regulation process (Figs. 1D).

At the S5 stage, 63 pathways were enriched with a statistically significant enrichment $p$-value. Plant hormone signal transduction (ko04075) and phenylpropanoid biosynthesis (ko00940) played active role during the entire process as well as up- and down-regulation processes, and the expression was significant. Glycerophospholipid metabolism (ko00564), starch and sucrose metabolism (ko00500) were active in the total process and the up-regulation process, which were not active in the down-regulation process. Glutathione metabolism (ko00480), drug metabolism - cytochrome P450 (ko00982), and metabolism of xenobiotics by cytochrome P450 (k000980) were triggered in the down -regulation process (Figs. 1E).

At the S6 stage, 31 pathways were enriched a with statistically significant enrichment $p$-value. Plant hormone signal transduction (ko04075), phenylpropanoid biosynthesis (ko00940), starch and sucrose metabolism (ko00500), glycolysis/gluconeogenesis (ko00010) played an active role during the entire process, the up- and down-regulation process, and the expression was significant. Valine, leucine and isoleucine degradation (ko00280) was triggered in the up-regulation process (Figs. 1F).

At the S7 stage, 39 pathways were enriched with a statistically significant enrichment p-value. Fatty acid metabolism (ko01212) was active during the entire process, but was not in the up- and down-regulation process. Plant hormone signal transduction (ko04075), phenylpropanoid biosynthesis (ko00940), starch and sucrose metabolism (ko00500) were active during the entire process and up-regulation process, while they were not in the down-regulation process. Tyrosine metabolism (ko00350) was active during the entire process and the down-regulation process, while it was not in the up-regulation process. Spliceosome (k0003040) was triggered in the up-regulation process, whereas MAPK signaling pathwayyeast (ko04011), as well as glycine, serine and threonine metabolism (ko00260) were triggered in the down -regulation process (Figs. 1G).

Summarizing the above KEGG pathways enriched during the flowering period, it was found that phenylpropanoid biosynthesis and flavonoid biosynthesis were active through the entire flowering process and nearly up-regulated at each stage, Peroxisome(ko04146), Carbon metabolism $\square k o 01200 \mathrm{Q}$ and Amino sugar and nucleotide sugar metabolism $\square k 000520 \square$ can be found in each period(Fig. 3A), and carotenoid biosynthesis was down-regulated from S1-6. The anthocyanin precursor, phenylalanine, was 
enhanced at the S1 phase; however, the synthesis of anthocyanin was weakened at the S5 and S6 phases (Table 2).

Table 2

Enriched KEGG pathways by up-regulated and down-regulated genes.

\begin{tabular}{|c|c|c|c|c|c|c|c|}
\hline KEGG Pathways & S1 & S2 & S3 & S4 & S5 & S6 & S7 \\
\hline Phenylpropanoid biosynthesis & $\uparrow \downarrow$ & $\uparrow \downarrow$ & $\uparrow \downarrow$ & $\uparrow$ & $\uparrow \downarrow$ & $\uparrow \downarrow$ & $\uparrow$ \\
\hline Carotenoid biosynthesis & $\downarrow$ & $\uparrow \downarrow$ & $\uparrow \downarrow$ & $\downarrow$ & & $\uparrow \downarrow$ & $\uparrow$ \\
\hline Flavonoid biosynthesis & $\uparrow$ & $\uparrow \downarrow$ & $\uparrow$ & $\uparrow$ & $\uparrow$ & $\uparrow$ & $\uparrow$ \\
\hline Phenylalanine metabolism & $\uparrow$ & & & & & & $\downarrow$ \\
\hline Phenylalanine, tyrosine and tryptophan biosynthesis & $\uparrow$ & & & & & & \\
\hline Anthocyanin biosynthesis & & & & & $\downarrow$ & $\downarrow$ & \\
\hline
\end{tabular}

\section{Gene Ontology (GO) analysis of differentially expressed genes}

Differentially expressed genes were annotated by Gene Ontology terms (Additional file 4: Table S4). Genes were classified into three GO categories, that is, Biological Process, Cellular Component, and Molecular Function. From the Biological Process, auxin-activated signaling pathway (G0:0009734), response to abscisic acid (G0:0009737), response to wounding (G0:0009611) were remarkable at the S1 stage. The negative regulation of the hydrogen peroxide metabolic process (G0:0010727), positive regulation of the receptor biosynthetic process (G0:0010870), cellular response to topologically incorrect protein (GO:0035967) were remarkable at the $S 2$ stage. The regulation of the transcription of nuclear large rRNA transcript from RNA polymerase I promoter (GO:1901836), nucleolar chromatin organization (GO:1990700), and regulation of superoxide anion generation (G0:0032928) were remarkable at the S3 stage.

The priming of the cellular response to stress (G0:0080136), cellular response to sucrose starvation (G0:0043617), and the cellulose metabolic process (G0:0030243) were remarkable at the S4 stage. The negative regulation of the gibberellin biosynthetic process (G0:0010373), guanosine tetraphosphate metabolic process (G0:0015969), and the regulation of the transcription of nuclear large rRNA transcript from RNA polymerase I promoter (G0:1901836) were remarkable at the S5 stage. The regulation of the transcription of nuclear large rRNA transcript from RNA polymerase I promoter (G0:1901836), nucleolar chromatin organization (G0:1990700), and the establishment of protein localization to endoplasmic reticulum membrane (G0:0097051) were remarkable at the S6 stage. The negative regulation of the 
extrinsic apoptotic signaling pathway in absence of ligand (GO:2001240), the phosphatidylinositol-3phosphate biosynthetic process (GO:0036092), and the regulation of the embryo sac egg cell differentiation (G0:0045694) were remarkable at the S7 stage (Figs. 2).

From the Cellular Component process, apoplast (G0:0048046), plant-type cell wall (G0:0009505), and cell wall (G0:0005618) were remarkable at the S1 stage. Inclusion body (G0:0016234), nitrite reductase complex $[\mathrm{NAD}(\mathrm{P}) \mathrm{H}](\mathrm{GO}: 0009344)$, and plasma membrane (G0:0005886) were remarkable at the $\mathrm{S} 2$ stage. Nucleolar chromatin (G0:0030874) vacuole (GO:0005773), and chloroplast (G0:0009507) were remarkable at the S3 stage. Anchored component of plasma membrane (G0:0046658), apoplast (GO:0048046), and plasma membrane (G0:0005886) were remarkable at the S4 stage. Nucleolar chromatin (G0:0030874), P4 peroxisome (G0:0019822), chloroplast (G0:0009507) were remarkable at the S5 stage. Nucleolar chromatin (G0:0030874), chloroplast (G0:0009507), vacuole (GO:0005773) were remarkable at the S6 stage. The DNA topoisomerase complex (ATP-hydrolyzing) (GO:0009330), nuclear ubiquitin ligase complex (GO:0000152), and TRAPPII protein complex (GO:1990071) were remarkable at the S7 stage (Figs. 2).

From the Molecular Function process, DNA binding transcription factor activity (G0:0003700), aspartictype endopeptidase activity (G0:0004190), hydrolase activity, and acting on ester bonds (G0:0016788) were remarkable at the $\mathrm{S} 1$ stage. Tau protein binding (G0:0048156), euchromatin binding (G0:1990188), and IAA-Phe conjugate hydrolase activity (G0:0010210) were remarkable at the S2 stage. IAA-Phe conjugate hydrolase activity (G0:0010210), IAA-Leu conjugate hydrolase activity (GO:0010211), and dehydroquinate synthase activity (GO:0102042) were remarkable at the S3 stage. Glucuronoxylan 4-0methyltransferase activity (GO:0030775), DNA binding transcription factor activity (GO:0003700), sequence-specific DNA binding (G0:0043565) were remarkable at the S4 stage. Euchromatin binding (GO:1990188), malonyl-CoA decarboxylase activity (GO:0050080), and glutamine-phenylpyruvate transaminase activity (G0:0047316) were remarkable in the S5 stage; euchromatin binding (GO:1990188), IAA-Phe conjugate hydrolase activity (GO:0010210), IAA-Leu conjugate hydrolase activity (GO:0010211) were remarkable at the S6 stage. Arylacetonitrilase activity (GO:0047428), euchromatin binding (GO:1990188), and calcium-independent phospholipase A2 activity (G0:0047499) were remarkable at the $\mathrm{S} 7$ stage (Figs. 2).

From seven periods, defense response to bacterium (G0:0042742) Dtranscription, DNA-templated

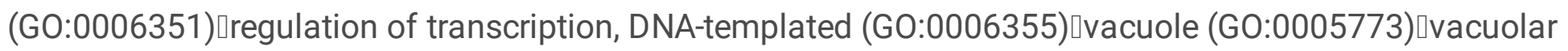
membrane (GO:0005774) extracellular region (GO:0005576)【chloroplast (GO:0009507) nucleus

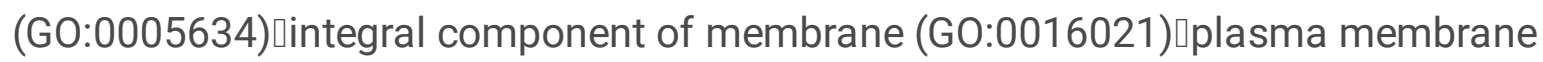

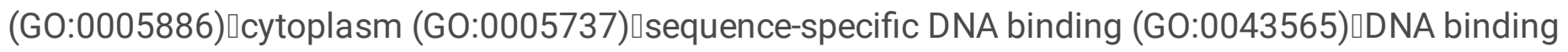
transcription factor activity (GO:0003700) $\square$ metal ion binding (GO:0046872) $\square$ ATP binding (GO:0005524) were found at the every period(Fig. 3B)

\section{Anthocyanin metabolism analysis of differentially expressed genes}


Sequencing results revealed that 114 unigenes were associated with anthocyanin metabolism, and 72 were significantly upregulated or downregulated across the seven flowering stages. The number of upregulated unigenes was highest at the $S 7$ stage, while the number of down-regulated unigenes was highest at the 55 stage (Fig. 4A). According to the analysis of TFs in anthocyanin metabolism, we obtained 47 genes relating to transcription factors in anthocyanin metabolism that belonged to 18 families, of which bHLH had the largest number, followed by GeBP (Additional file 5: Table S5, Fig. 4B). According to the anthocyanin heatmap analysis, the expression of genes at S7 was different from that at other stages, with the highest up-regulated expression. The expression of genes at $\mathbf{S} 6$ was similar to that at S5, while those at S4, S3, S2, and S1 were similar, among which the expression of genes in S1 and S2 were the most similar (Fig. 4C).

\section{qRT-PCR verification}

To validate the accuracy and repeatability of our RNA-Seq data, the current study selected six genes for quantitative real-time polymerase chain reaction (qRT-PCR) analysis, with gene-specific primers designed using Primer software (version 5.0), as shown in Additional file 6: Table S6. As shown in Fig. 5, the results of qRT-PCR indicated that most of these genes had expression patterns that correlated with the RNA-Seq data, which confirmed the reliability of our data. The genes LjDFR (TRINITY_DN37612_c0_g1_i2_29), LjABCB1 (TRINITY_DN23592_C0_g2_i2_8), LjMYC6 (TRINITY_DN17985_C0_g1_i2_8), LjDDB2 (TRINITY_DN25019_c0_g2_i6_8), and LjANS (TRINITY_DN27734_c0_g1_i1_12) rapidly increased during the first three stages. However, the expression of only LjF3'5'H (TRINITY_DN27086_c0_g1_i1_29) was significantly down-regulated at S5, which was consistent with anthocyanin accumulation.

\section{Discussion}

The biological regulation of flower color had garnered much attention from researchers, particularly in terms of ornamental plant research [11]. This present study focused on a new variety of L. japonica (Yujin no. 2). There are a variety of designs and colors for 'Yujin 2' that has a high ornamental value, which may play a significant role in landscaping [9]. It is very important to regulate anthocyanin synthesis in $L$. japonica breeding [22]. With respect to molecular breeding, the identification of the key genes involved in anthocyanin synthesis will enable the overexpression or knockout of these genes to facilitate color modification [23]. Therefore, this study began with the aim of identifying the essential genes that regulated the alteration of flower color and subsequently exploring the potential molecular mechanisms behind the development of flower color.

However, little evidence has been reported as relates to $L$. japonica petal color. The current study results provide a foundation for research into petal color mechanisms at the transcriptome level. Transcriptome analysis based on an EST sequencing dataset illustrated the characteristics of the carnation transcriptome and revealed that carotenoid, chlorophyll, and anthocyanin biosynthesis played roles in the regulation of carnation flower color [24]. 
Correlation analysis showed that the changes in Paeonia flower color, from coral to pink to pale yellow were due to a significant decrease in anthocyanin content [25]. It was deduced that the disequilibrium of expression levels in flavonol synthases and dihydroflavonol-4-reductases resulted in different levels of anthocyanin accumulation and coloration in white and pink tea flowers via metabolome and transcriptome analysis [26]. Besides, the transcriptome dataset assisted with the establishment of linkages between gene expression and other aspects, including the phenotypical, biochemical and metabolic features of Meconopsis petal development [10].

Petal color change is a significant feature of gene expression. As a whole, the number of differentially expressed genes at each flowering development stage was variable, which revealed that transcriptome expression changes were more active during color alteration. For example, when the petal color changed from purple-red (S1) to red (S2), 15804 genes were differentially expressed; while only 2033 genes changed from S2 to S3, when the petal color at both were red. Further, when the petal colors were red, but gradually became lighter from the three-green period (S2) to the great white period (S4), the number of differentially expressed genes were eventually lower, which were 21159,19126 , and 11153 , respectively. Besides, gene expression changes occurred when the petal color transitioned from red (S4), to the outer part being red and the inner part being white (S5), where 6073 genes were up-regulated from the bud stage to cracking stage (Fig. 2).

Petal color phenotypes result from plant pigment metabolism [13]. Anthocyanin, flavonoid, and alkaloid are well-known plant pigments that play active roles in the regulation of flower color [11]. Flavonoids are involved in the production of red, pink, purple and blue in plants and are widely distributed in petals [27]. Additionally, and most importantly, flavonoid accumulation in a spontaneous cotton mutant resulted in red coloration and enhanced disease resistance [28]. Anthocyanins are a group of water-soluble pigments that confer the blue, purple and red color to many fruits [29]. Carotenoids primarily affect yellow, orange and red coloration [30]. To identify the potential mechanisms that regulate the modification of flowering colors, the current study annotated differentially expressed genes via KEGG pathway analysis and Gene Ontology.

In the pathway analysis, several pigment metabolism pathways were highlighted, including plant hormone signal transduction (ko04075), phenylpropanoid biosynthesis (ko00940), anthocyanin biosynthesis (ko00942), flavonoid biosynthesis (ko00941), and carotenoid biosynthesis (ko00906). Furthermore, plant hormone signal transduction, phenylpropanoid biosynthesis, and flavonoid biosynthesis were active throughout the flowering process and nearly up-regulated at each stage. These results corroborated the findings of several previous investigations into the linkages between petal color and plant pigment. Previous studies found that the flowers of 'Yujin 2' were red due to their higher carotenoid and flavonoid content in contrast to 'Damaohua' at all developmental stages [9].

However, the results of our study revealed that carotenoid biosynthesis was down-regulated throughout S1-6, which was different from those of previous studies to some extent, due to the formation of mutants that varied with sampling times. Phenylalanine is an important anthocyanin biosynthesis pathway [13]. In 
this study, phenylalanine synthesis was enhanced at the $\mathrm{S} 1$ phase, and anthocyanin synthesis was weakened at S5 and S6. This result may be consistent with the changes of 'Yujin 2' petal color from red (S1) to white (S5) and gold (S6).

The results obtained in the current study revealed that the phenylpropanoid biosynthetic process (GO:0009699), carotenoid biosynthetic process (GO:0016117), and anthocyanin 5-0-glucoside-4"'-0malonyltransferase activity (G0:0102801) were triggered. Anthocyanin is the stable configuration of anthocyanidins that is always combined with a sugar moiety [31], which may explain the active glucoserelated metabolism pathways in our research.

GO annotation also supported the KEGG evidence. Furthermore, GO annotation showed that plasma membrane (G0:0005886) was active throughout the flowering process. Chloroplast (G0:0009507) and vacuole (G0:0005773) were active through most of the flowering processes, and could be explained by the results of previous experiments. The colors of plants are primarily codetermined by the different distributions of chlorophyll, carotenoid, flavonoid, and betaine, which exist in the cytoplasms or vacuoles of plants [9]. Moreover, $\mathrm{GO}$ analysis revealed that environment-related responses (such as to cold) were triggered (GO:0009409).

Generally, the binding of upstream transcription factors, initiation of gene transcription and expression, final synthesis of anthocyanins, and coloration of fruits and peels are closely related to the expression of structural genes in the anthocyanin biosynthetic pathway [19]. At present, MYB, BHLH and WD40 are the three major transcription factors currently known. Anthocyanin biological regulation includes singlestructure gene regulation, two-gene interaction regulation, and some MBW (MYB-bHLH-WD40) complexes regulate anthocyanin accumulation [16].

In the first three stages of flower development, LjDFR, LjABCB1, LjMYC6, LjDDB2, and LjANS genes increased rapidly. However, only the expression of LjF3'5'H was significantly down-regulated at S5, which was consistent with the accumulation of anthocyanin. The expression of other transcription factors did not increase during anthocyanin accumulation, which may not be directly related to anthocyanin synthesis. To verify the RNA-Seq results, we randomly selected six genes and tested their expression patterns via qPCR, which revealed the same expression trend with the sequencing results.

\section{Conclusions}

In summary, this study developed a transcriptome profile of flower color generation for $L$. japonica as well as annotated unigene sets of seven anthesis phases. Bioinformatics analysis revealed a number of distinct clues in regard to color development mechanisms. Further, candidate genes and potential pathways were obtained, which provided a foundation for further research at the transcriptome level. However, the current results still did not fully elucidate the overall regulatory mechanisms of plant pigments and the formation of the red color for L. japonica. Therefore, future studies relating to the current topic, such as transcript regulation, protein expression, and biochemical metabolism, are 
recommended. Candidate key genes involved in anthocyanin metabolism should also be further investigated.

\section{Methods}

\section{Plant material preparation}

Five year old 'Damaohua' and 'Yujin 2' were planted in the resource garden of the College of Life Sciences,

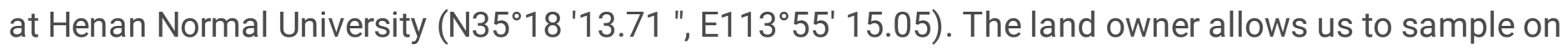
their private land. Professor Jingyuan Li identifies 'Damaohua' and 'Yujin 2' as honeysuckle [9] (Fig. 6). Our research group collected fresh flower buds of of 'Damaohua' and 'Yujin 2' in seven periods: young bud stage (S1), three green stage (S2), two white stage (S3), great white stage (S4), silver stage (S5), golden stage (S6), fade stage (S7). During sample collection, the flowers were combined and regarded as one biological replicate representing each stage, and three independent replicates were prepared. Partial flower materials were immediately frozen in liquid nitrogen following collection and stored at $-80^{\circ} \mathrm{C}$.

\section{RNA isolation and library construction}

The total RNA was extracted by mirVana miRNA Isolation Kit (Ambion) [32]. RNA integrity was evaluated using the Agilent 2100 Bioanalyzer (Agilent Technologies, Santa Clara, CA, USA), and samples with the RNA Integrity Number (RIN) $\geq 7$ were subjected to subsequent analysis. Separate mRNA from total RNA with oligonucleotide beads (dT) and add fragment buffer to cut it into short fragments, and then use random hexamer primers as templates to synthesize first strand cDNA. The library was constructed according to the manufacturer's instructions for TruSeq chain mRNA LTSample Prep Kit (Illumina, San Diego, California, USA).

\section{Sequencing, de novo assembly and annotation}

The above libraries were sequenced using an Illumina HiSeq X Ten sequencer (Illumina Inc., USA) and $150 \mathrm{bp}$ paired-end reads were generated. The preparation of the cDNA library and sequencing were performed at Shanghai OE Biotech. Co., Ltd., Shanghai, China, and raw data (raw reads) were processed using Trimmomatic [33]. The reads containing ploy- $\mathrm{N}$ and low quality reads were removed to obtain clean reads. Following the removal of adaptor and low quality sequences, the clean reads were assembled into expressed sequence tag clusters (contigs) and assembled de novo into transcripts using Trinity [34] (version: trinityrnaseq_r20131110) through a paired-end method. The longest transcript was selected as a unigene based on the similarity and length of a sequence for subsequent analysis.

The function of the unigenes was annotated to the NCBI non-redundant protein (NR), Clusters of Orthologous Groups (COG) for eukaryotic complete genomes, Gene Ontology (GO), Swiss-Prot, and evolutionary genealogy of genes: Non-supervised Orthologous Groups (eggNOG) and Kyoto Encyclopedia of Genes and Genomes (KEGG) databases by diamond software, and mapped to Pfam databases by HMMER. The search was conducted using Blastx with a threshold E-value cut-off of 1e-5. Unigene 
quantification, analysis of differentially expressed unigenes (DEGs), cluster analysis, GO and KEGG enrichment FPKM [35], and read count values of each unigene were calculated using bowtie2 [36]and eXpress[37].

We use the DESeq function to estimate the size factor and the nbinom test to determine DEG [38]. The threshold value of $\mathrm{P}<0.05$ and multiple change $>2$ or multiple change $<0.5$ was set as the significant difference threshold.To explore the transcriptional expression patterns of DEGs, cluster analysis was performed. We performed GO analysis and KEGG pathway enrichment analysis for DEGs based on hypergeometric distribution of R.

\section{Expression analysis of transcription factors (TFs)}

PlantTFDB (http://planttfdb.cbi.pku.edu.cn/index.php) contains sequences of 58 plant transcription factor families from 165 plants, and is a plant transcription factor database [39]. Blastx was used to compare the sequence of unigenes with transcription factor database, and the best gene with $E$ value less than $1 \mathrm{e}-5$ was selected as annotation information of unigene. Candidates that contained DNA binding domains were recognized by GO annotation for the final TF identification.

Differentially expressed TFs (DETFs) between samples were identified using the value of Fragments Per Kilobase of transcript Per Million fragments mapped (FPKM) with $\mid \log 2$ (fold change) $\mid>1$, $p$ value $\leq 0.05$ and q value $\leq 0.05$ (Bolger et al. 2014). Multiexperiment Viewer software (v4.9) was employed to exhibit the expression profiles of DETFs by clustering [34] and the DETFs were clustered via the K-means method. Using the total TFs in L. japonica as reference, GO enrichment analysis of DETFs was performed using Agrigo (http:// bioinfo.cau.edu.cn/agriGO/) with the hypergeometric test and FDR cut-off of 0.05 [40].

\section{qRT-PCR}

The same RNA samples used for the RNA-seq experiments were employed for qRT-PCR. We use NanoDrop 2000 spectrophotometer to measure RNA yield (Thermo Scientific, USA), whereas the integrity was evaluated using agarose gel electrophoresis stained with ethidium bromide. Quantification was performed using a two-step reaction process: reverse transcription (RT) and PCR. Each sample was run in triplicate for analysis. At the end of the PCR cycles, melting curve analysis was performed to validate the specific generation of the expected PCR product. Quantification was performed using the $2-\triangle \Delta C T$ method, and data were normalized to the ACT2/7 transcript [41]. The sequences of the primers used are listed in Additional file 1: Table S1.

\section{Statistical analysis}

Significant differences were calculated using a one-way ANOVA analysis with a Turkey test and a significance level at $p \leq 0.05$ and $p \leq 0.01$ in SPSS 19.0 software [9]. All expression analyses were repeated three times. The reported value represents the arithmetic mean of three replicates. Data was expressed as a mean plus or minus a standard deviation (mean \pm SD).

\section{Abbreviations}

Page 13/23 
L. japonica Lonicera japonica Thunb

DEGs differentially expressed genes

CGA chlorogenic acid

CHS chalcone synthase,

F3H flavanone 3-hydroxylase,

DFR dihydroflavonol-4-reductase,

ANS anthocyanidin synthase,

UFGT UDP-glucose flavonoid 3-O-glucosy/transferase

\section{Declarations}

\section{Ethics approval and consent to participate}

Not applicable

\section{Consent for publication}

Not applicable

\section{Availability of data and material}

All data generated or analyzed for this study are included in this article and its supplementary information files.

\section{Competing interests}

The authors declare that they have no competing interests

\section{Funding}

The work was carried out under the auspices of the Public Health Services of Traditional Chinese Medicine in 2017 [(2017)66] and Henan Province Enterprise Technology Innovation Guidance Special Project (172107000031).

\section{Authors' contributions}

LJ.J. designed the research methodology, C C.F. and L Y. performed data analysis and wrote the article, and Y C.L,MJ.X. and C T prepared the plant samples. All authors have read and approved the manuscript. 
At the point of finishing this paper, l'd like to express my sincere thanks to all those who have lent me hands in the course of my writing this paper.

\section{References}

1. Shang X, Pan H, Li M, Miao X, Ding H. Lonicera japonica Thunb.: ethnopharmacology, phytochemistry and pharmacology of an important traditional Chinese medicine. J Ethnopharmacol. 2011;138(1):1-21. doi:10.1016/j.jep.2011.08.016.

2. Hsu HF, Hsiao PC, Kuo TC, Chiang ST, Chen SL, Chiou SJ, Ling XH, Liang MT, Cheng WY, Houng JY. Antioxidant and anti-inflammatory activities of Lonicera japonica Thunb. var. sempervillosa Hayata flower bud extracts prepared by water, ethanol and supercritical fluid extraction techniques. Ind Crop Prod. 2016;89:543-9. doi:10.1016/j.indcrop.2016.05.010.

3. .3 Wang DY, Zhao XM, Liu YL. (2017) Hypoglycemic and hypolipidemic effects of a polysaccharide from flower buds of Lonicera japonica in streptozotocin-induced diabetic rats. International journal of biological macromolecules 102:396-404. doi:10.1016/j.ijbiomac.2017.04.056.

4. Tian J, Che HL, Ha D, Wei YP, Zheng SY. Characterization and anti-allergic effect of a polysaccharide from the flower buds of Lonicera japonica. Carbohyd Polym. 2012;90(4):1642-7. doi:10.1016/j.carbpol.2012.07.044.

5. Ryu KH, Rhee HI, Kim JH, Yoo H, Lee BY, Um KA, Kim K, Noh JY, Lim KM, Chung JH. Anti-Inflammatory and Analgesic Activities of SKLJI, a Highly Purified and Injectable Herbal Extract of Lonicera japonica. Biosci Biotech Bioch. 2010;74(10):2022-8. doi:10.1271/bbb.100279.

6. Han J, Lv QY, Jin SY, Zhang TT, Jin SX, Li XY, Yuan HL. Comparison of anti-bacterial activity of three types of di-O-caffeoylquinic acids in Lonicera japonica flowers based on microcalorimetry. Chin $\mathrm{J}$ Nat Medicines. 2014;12(2):108-13. doi:10.1016/S1875-5364(14)60017-0.

7. Ning W, Peng X, Ma LY, Cui L, Lu XP, Wang J, Tian JK, Li XM, Wang W, Zhang L. Enhanced secondary metabolites production and antioxidant activity in postharvest Lonicera japonica Thunb. in response to UV radiation. Innov Food Sci Emerg. 2012;13:231-43. doi:10.1016/j.ifset.2011.10.005.

8. Wang Z, Clifford MN, Sharp P. Analysis of chlorogenic acids in beverages prepared from Chinese health foods and investigation, in vitro, of effects on glucose absorption in cultured Caco-2 cells. Food Chem. 2008;108(1):369-73. doi:10.1016/j.foodchem.2007.10.083.

9. Li JJ, Lian XY, Ye CL, Wang L. Analysis of Flower Color Variations at Different Developmental Stages in Two Honeysuckle (Lonicera Japonica Thunb.) Cultivars. Hortscience. 2019;54(5):779-82. doi:10.21273/Hortsci13819-18.

10. Qu Y, Ou Z, Yang FS, Wang S, Peng J. The study of transcriptome sequencing for flower coloration in different anthesis stages of alpine ornamental herb (Meconopsis 'Lingholm'). Gene. 2019;689:2206. doi:10.1016/j.gene.2018.12.017.

11. Zhao D, Tao J. Recent advances on the development and regulation of flower color in ornamental plants. Front Plant Sci. 2015;6:261. doi:10.3389/fpls.2015.00261. 
12. Yoshida K, Mori M, Kondo T. Blue flower color development by anthocyanins: from chemical structure to cell physiology. Nat Prod Rep. 2009;26(7):884-915. doi:10.1039/b800165k.

13. Tanaka Y, Sasaki N, Ohmiya A. Biosynthesis of plant pigments: anthocyanins, betalains and carotenoids. Plant J. 2008;54(4):733-49. doi:10.1111/j.1365-313X.2008.03447.x.

14. Tanaka Y, Brugliera F, Kalc G, Senior M, Dyson B, Nakamura N, Katsumoto Y, Chandler S. Flower Color Modification by Engineering of the Flavonoid Biosynthetic Pathway: Practical Perspectives. Biosci Biotech Bioch. 2010;74(9):1760-9. doi:10.1271/bbb.100358.

15. Sadilova E, Stintzing FC, Carle R. Anthocyanins, colour and antioxidant properties of eggplant (Solanum melongena L.) and violet pepper (Capsicum annuum L.) peel extracts. Z Naturforsch C. 2006;61(7-8):527-35.

16. Yang Y, Cui BH, Tan ZW, Song BX, Cao HN, Zong CW. (2018) RNA sequencing and anthocyanin synthesis-related genes expression analyses in white-fruited Vaccinium uliginosum. Bmc Genomics 19. doi:ARTN 93010.1186/s12864-018-5351-0.

17. Gao LX, Yang HX, Liu HF, Yang J, Hu YH. (2016) Extensive Transcriptome Changes Underlying the Flower Color Intensity Variation in Paeonia ostii. Frontiers in Plant Science 6. doi:ARTN $120510.3389 /$ fpls.2015.01205.

18. Tripathi AM, Niranjan A, Roya S. Global gene expression and pigment analysis of two contrasting flower color cultivars of Canna. Plant Physiol Bioch. 2018;127:1-10. doi:10.1016/j.plaphy.2018.03.008.

19. Zhao L, Gao LP, Wang HX, Chen XT, Wang YS, Yang H, Wei CL, Wan XC, Xia T. The R2R3-MYB, bHLH, WD40, and related transcription factors in flavonoid biosynthesis. Funct Integr Genomic. 2013;13(1):75-98. doi:10.1007/s10142-012-0301-4.

20. Bi H, Luang S, Li Y, Bazanova N, Morran S, Song Z, Perera MA, Hrmova M, Borisjuk N, Lopato S. Identification and characterization of wheat drought-responsive MYB transcription factors involved in the regulation of cuticle biosynthesis. J Exp Bot. 2016;67(18):5363-80. doi:10.1093/jxb/erw298.

21. Zheng J, Wu H, Zhu HB, Huang CY, Liu C, Chang YS, Kong ZC, Zhou ZH, Wang GW, Lin YJ, Chen H. Determining factors, regulation system, and domestication of anthocyanin biosynthesis in rice leaves. New Phytol. 2019;223(2):705-21. doi:10.1111/nph.15807.

22. Wiczkowski W, Szawara-Nowak D, Topolska J. Changes in the content and composition of anthocyanins in red cabbage and its antioxidant capacity during fermentation, storage and stewing. Food Chem. 2015;167:115-23. doi:10.1016/j.foodchem.2014.06.087.

23. Tasaki K, Higuchi A, Watanabe A, Sasaki N, Nishihara M. Effects of knocking out three anthocyanin modification genes on the blue pigmentation of gentian flowers. Sci Rep. 2019;9(1):15831. doi:10.1038/s41598-019-51808-3.

24. Tanase K, Nishitani C, Hirakawa H, Isobe S, Tabata S, Ohmiya A, Onozaki T. Transcriptome analysis of carnation (Dianthus caryophyllus L.) based on next-generation sequencing technology. BMC Genom. 2012;13:292. doi:10.1186/1471-2164-13-292. 
25. Guo L, Wang Y, da Silva JAT, Fan Y, Yu X. Transcriptome and chemical analysis reveal putative genes involved in flower color change in Paeonia 'Coral Sunset'. Plant Physiol Biochem. 2019;138:130-9. doi:10.1016/j.plaphy.2019.02.025.

26. Zhou C, Mei X, Rothenberg DO, Yang Z, Zhang W, Wan S, Yang H, Zhang L. (2020) Metabolome and Transcriptome Analysis Reveals Putative Genes Involved in Anthocyanin Accumulation and Coloration in White and Pink Tea (Camellia sinensis) Flower. Molecules 25 (1). doi:10.3390/molecules25010190.

27. Williams CA, Grayer RJ. Anthocyanins and other flavonoids. Nat Prod Rep. 2004;21(4):539-73. doi:10.1039/b311404j.

28. Long L, Liu J, Gao Y, Xu FC, Zhao JR, Li B, Gao W. Flavonoid accumulation in spontaneous cotton mutant results in red coloration and enhanced disease resistance. Plant Physiol Biochem. 2019;143:40-9. doi:10.1016/j.plaphy.2019.08.021.

29. Fang J. Classification of fruits based on anthocyanin types and relevance to their health effects. Nutrition. 2015;31(11-12):1301-6. doi:10.1016/j.nut.2015.04.015.

30. Rosas-Saavedra C, Stange C. Biosynthesis of Carotenoids in Plants: Enzymes and Color. Subcell Biochem. 2016;79:35-69. doi:10.1007/978-3-319-39126-7_2.

31. Veitch NC, Grayer RJ. Flavonoids and their glycosides, including anthocyanins. Nat Prod Rep. 2011;28(10):1626-95. doi:10.1039/c1np00044f.

32. Yang B, Guan Q, Tian J, Komatsu S. Transcriptomic and proteomic analyses of leaves from Clematis terniflora DC. under high level of ultraviolet-B irradiation followed by dark treatment. Journal of proteomics. 2017;150:323-40. doi:10.1016/j.jprot.2016.10.001.

33. Bolger AM, Lohse M, Usadel B. Trimmomatic: a flexible trimmer for Illumina sequence data. Bioinformatics. 2014;30(15):2114-20. doi:10.1093/bioinformatics/btu170.

34. Grabherr MG, Haas BJ, Yassour M, Levin JZ, Thompson DA, Amit I, Adiconis X, Fan L, Raychowdhury R, Zeng QD, Chen ZH, Mauceli E, Hacohen N, Gnirke A, Rhind N, di Palma F, Birren BW, Nusbaum C, Lindblad-Toh K, Friedman N, Regev A. Full-length transcriptome assembly from RNA-Seq data without a reference genome. Nat Biotechnol. 2011;29(7):644-U130. doi:10.1038/nbt.1883.

35. Trapnell C, Williams BA, Pertea G, Mortazavi A, Kwan G, van Baren MJ, Salzberg SL, Wold BJ, Pachter L. Transcript assembly and quantification by RNA-Seq reveals unannotated transcripts and isoform switching during cell differentiation. Nat Biotechnol. 2010;28(5):511-5. doi:10.1038/nbt.1621.

36. Langmead B, Salzberg SL. Fast gapped-read alignment with Bowtie 2. Nat Methods. 2012;9(4):357U354. doi:10.1038/Nmeth.1923.

37. Roberts A, Pachter L. Streaming fragment assignment for real-time analysis of sequencing experiments. Nat Methods. 2013;10(1):71-3. doi:10.1038/nmeth.2251.

38. Gupta R, Dewan I, Bharti R, Bhattacharya A. (2012) Differential Expression Analysis for RNA-Seq Data. ISRN Bioinform 2012:817508. doi:10.5402/2012/817508.

39. Jin J, Tian F, Yang DC, Meng YQ, Kong L, Luo J, Gao G. PlantTFDB 4.0: toward a central hub for transcription factors and regulatory interactions in plants. Nucleic Acids Res. 2017;45(D1):D1040-5. 
doi:10.1093/nar/gkw982.

40. Mortazavi A, Williams BA, McCue K, Schaeffer L, Wold B. Mapping and quantifying mammalian transcriptomes by RNA-SEq. Nat Methods. 2008;5(7):621-8. doi:10.1038/nmeth.1226.

41. Livak KJ, Schmittgen TD. Analysis of relative gene expression data using real-time quantitative PCR and the 2(-Delta Delta C(T)) Method. Methods. 2001;25(4):402-8. doi:10.1006/meth.2001.1262.

\section{Figures}

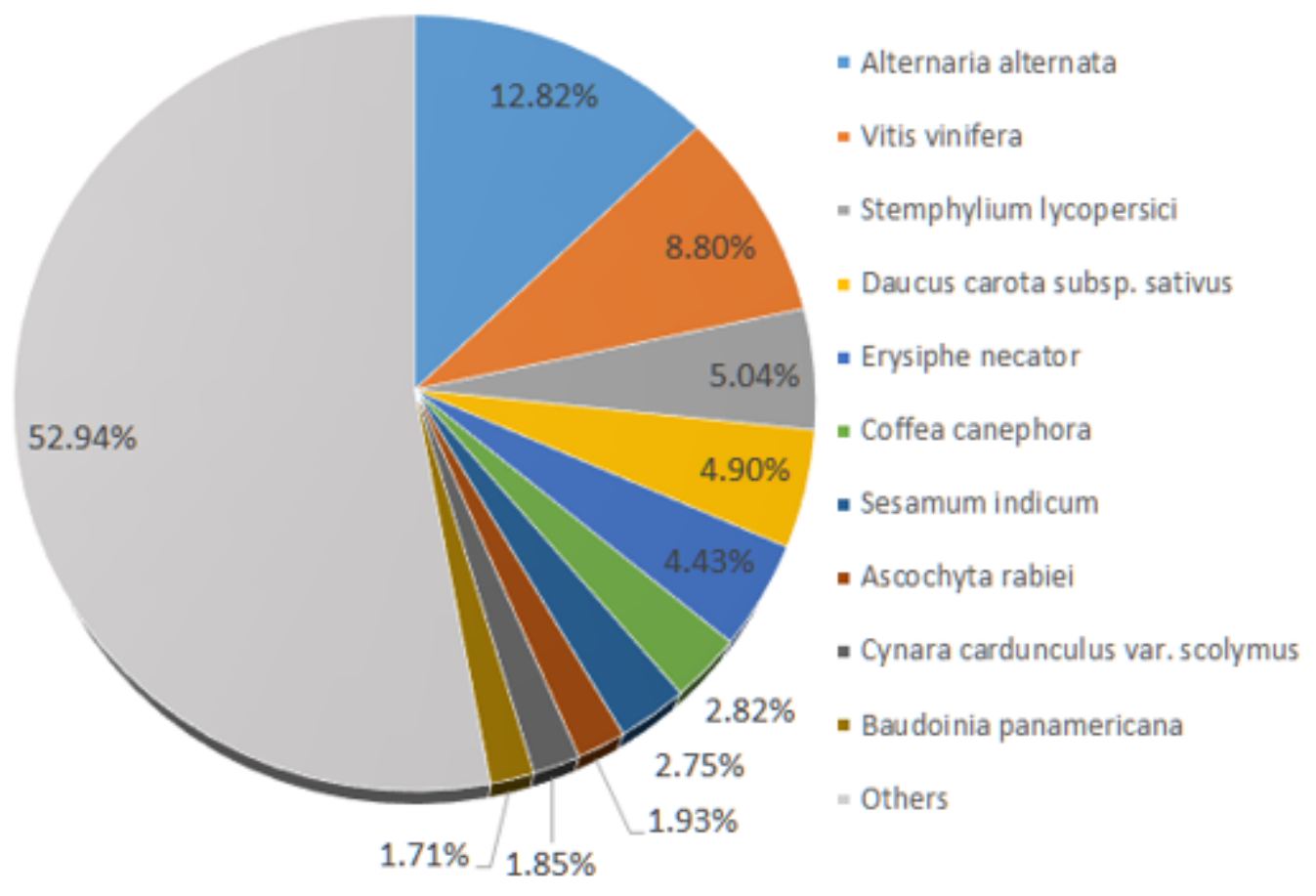

Figure 1

Species taxonomy based on the Nucleotide (NR) database. 


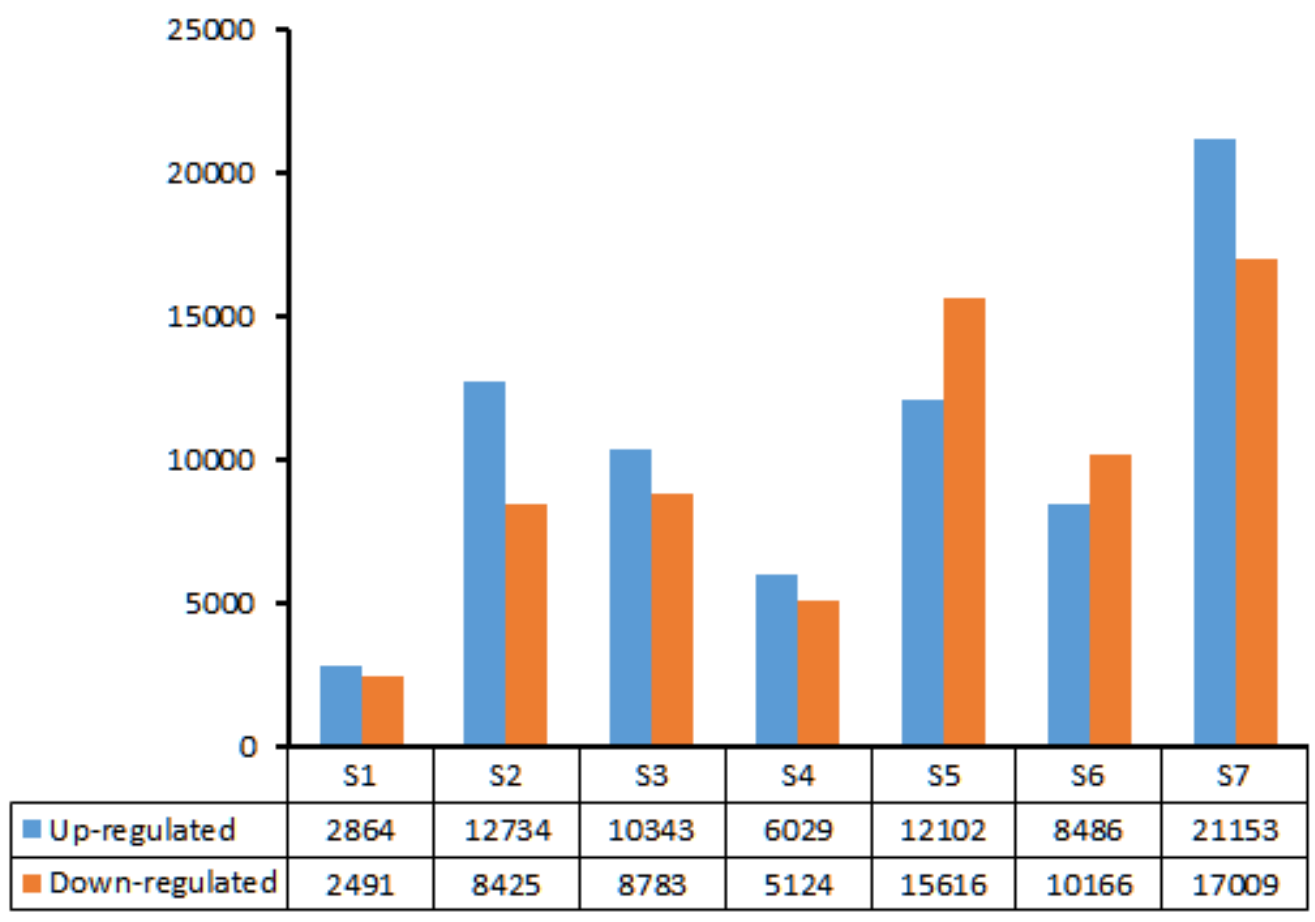

\section{Figure 2}

Number of up-regulated and down-regulated genes across seven flowering stages.

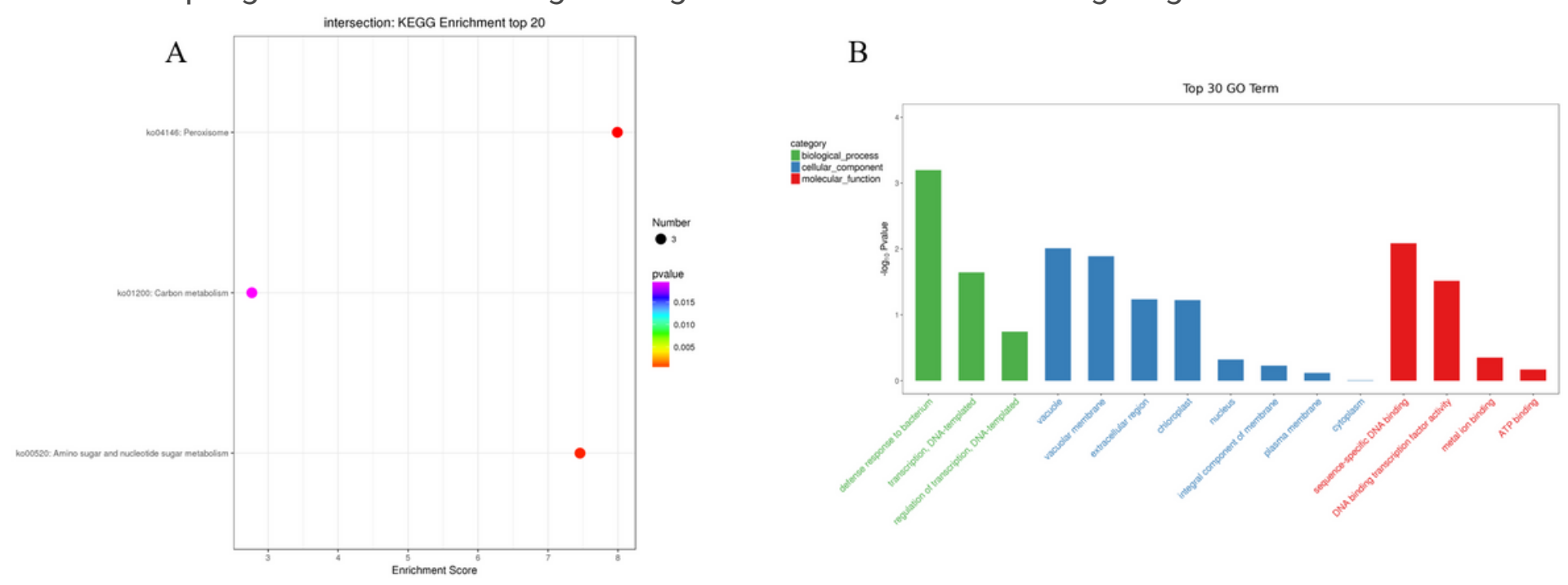

\section{Figure 3}

Enriched KEGG pathway that existed in seven periods $₫ \mathrm{~B}$ : Enriched $\mathrm{GO}$ pathway that existed in seven periods. 

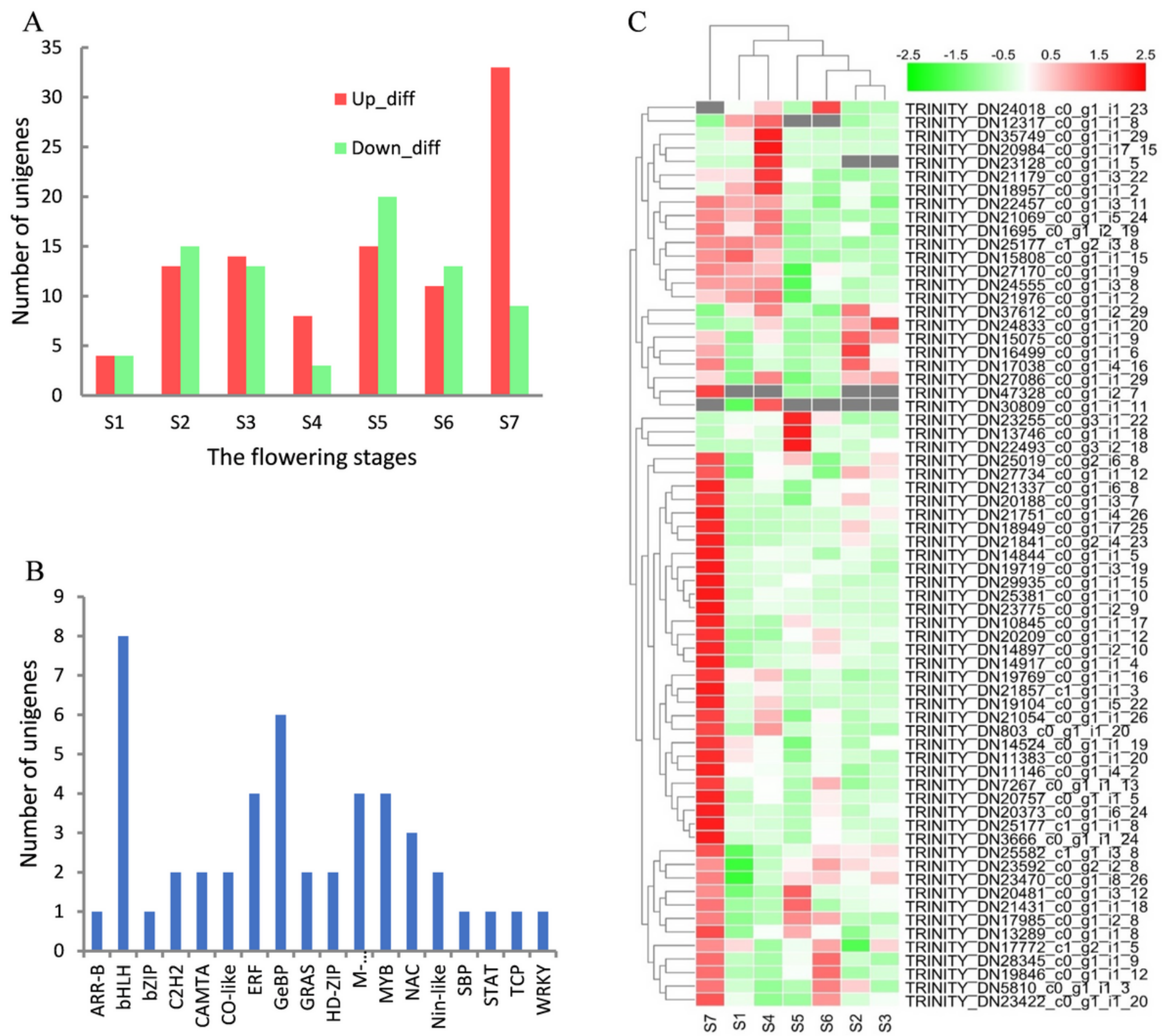

Family

\section{Figure 4}

Identification of potential unigenes associated with anthocyanin metabolism, and their expression levels at different stages of L. japonica. A: The numbers of upregulated or downregulated across the seven flowering stages; $\mathrm{B}$ : DEGs related to TFs in anthocyanin development; $\mathrm{C}$ : Transcript expression analysis for unigenes associated with anthocyanin development. Changes in expression level are represented by color; green indicates a lower expression level, whereas red indicates a higher expression level. 

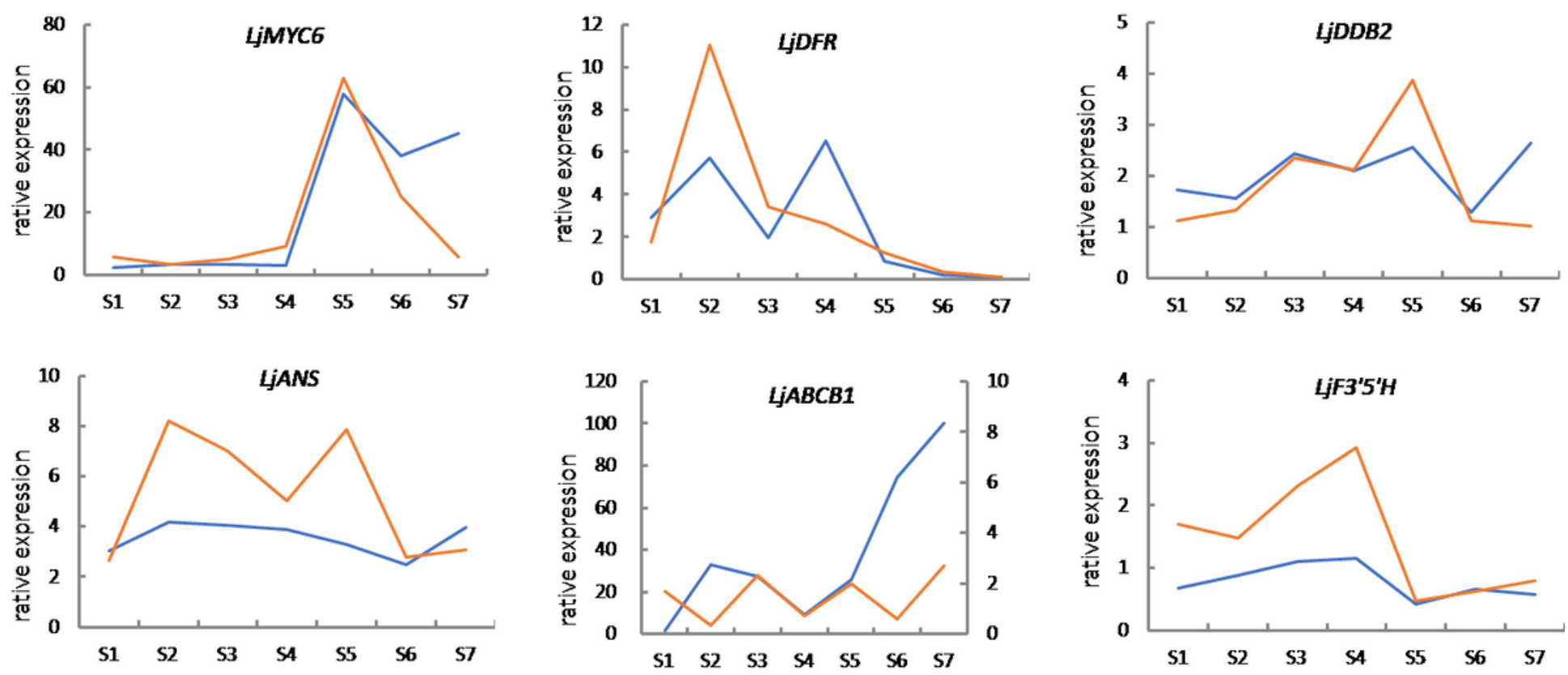

Figure 5

Real-time qPCR validation of six unigenes involved in anthocyanin metabolism. The orange line indicates qPCR results, blue line indicates RNA-Seq results. 


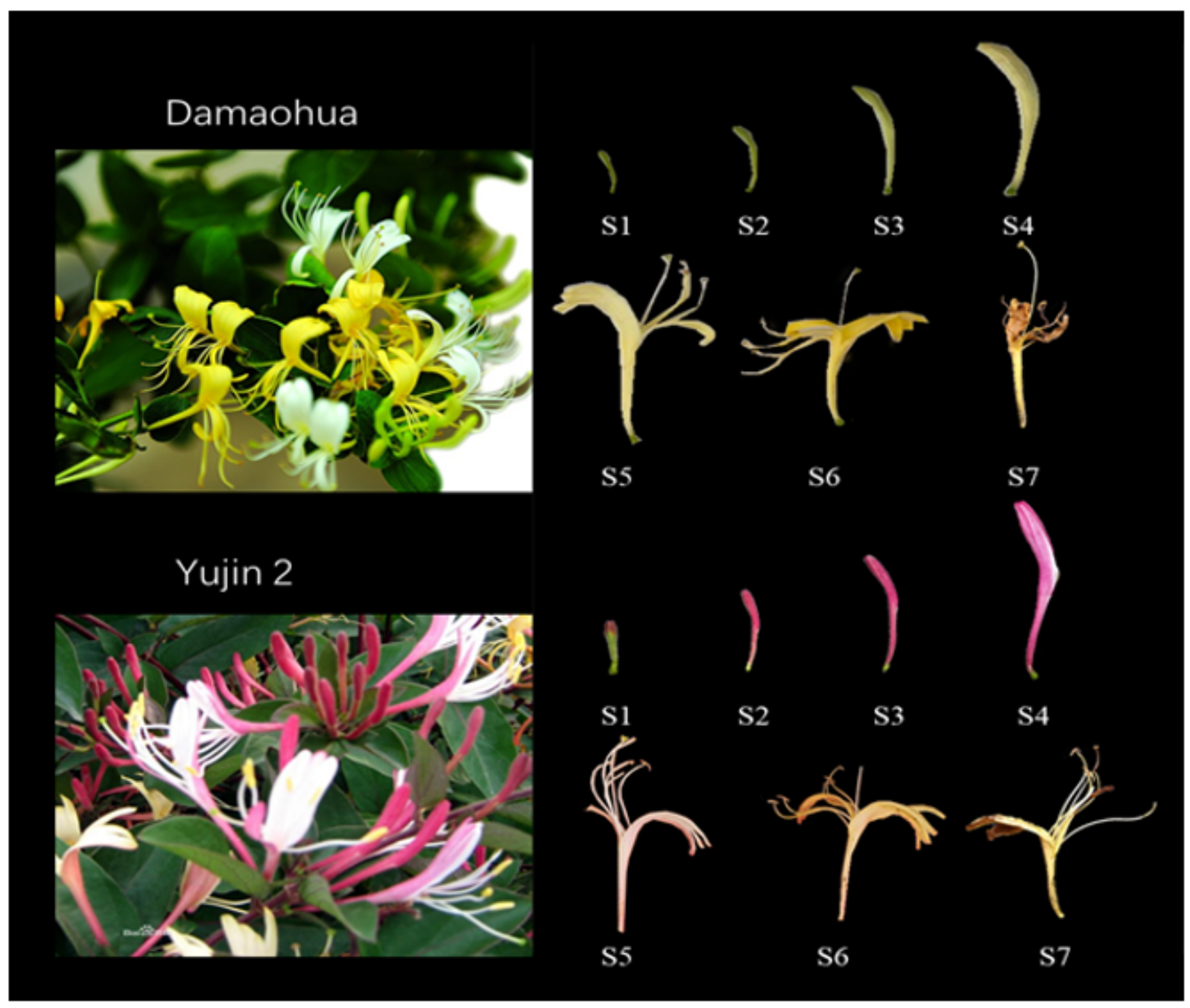

Figure 6

The morphology of flowers at different developmental stages in two Lonicera Japonica Thunb. 

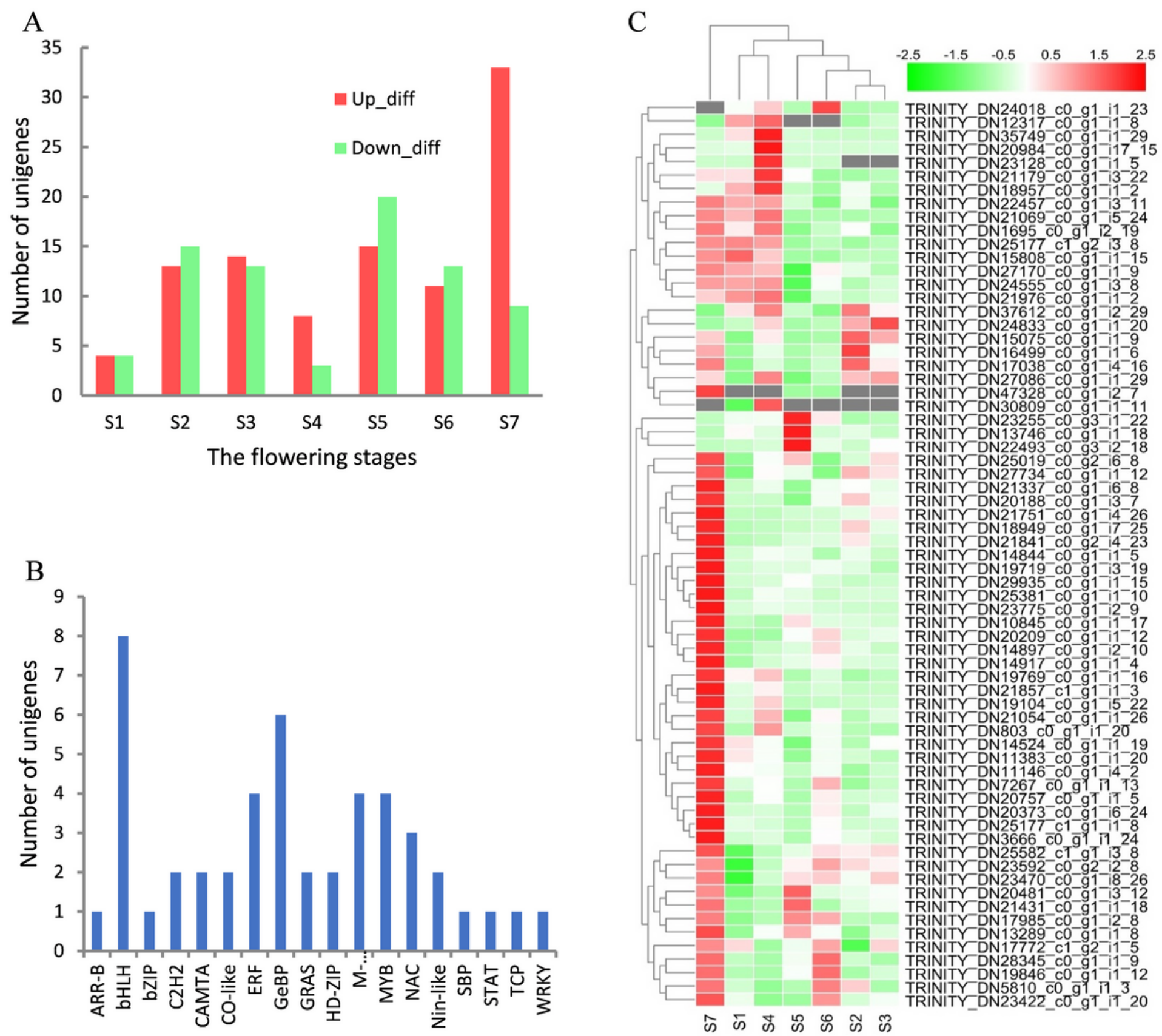

Family

Figure 7

\section{Supplementary Files}

This is a list of supplementary files associated with this preprint. Click to download.

- Additionalfile.xlsx 